\title{
A irracionalidade e transcendência de certos logaritmos
}

\author{
Ronald Pinto Liliana da Costa
}

\begin{abstract}
Resumo
Neste artigo exibimos algumas demonstrações de irracionalidade de certos logaritmos. A importância deste fato relaciona-se com a caracterização dos números irracionais pela representação decimal infinita não-periódica. Tanto nas antigas tábuas de logaritmos como nas calculadoras eletrônicas os valores dos logaritmos são representados na forma de uma expressão decimal finita. Isto pode gerar a falsa impressão de que se tratam necessariamente de números racionais. Ao contrário de diversos textos e artigos sobre o assunto, não ficamos restritos ao logaritmo decimal. Com efeito, mostramos uma condição suficiente para o número $\log _{b} a$ ser irracional, onde $a>0$ e $b>1$ são inteiros e também um critério que estabelece a irracionalidade de $\log _{b} a$ quando $b>1$ for livre de quadrados. Mostramos por fim, lançando mão do Teorema de Gelfond-Schneider, algumas provas da transcendência de certos logaritmos e tratamos de logaritmos de números reais não-inteiros.
\end{abstract}

Palavras-chave: números irracionais, logaritmos, Teorema Fundamental da Aritmética, números transcendentes.

\section{Abstract}

In this article we show some demonstrations of irrationality of certain logarithms. The importance of this fact is related to the characterization of the irrational numbers by the infinite non-periodic decimal representation. In both the old logarithmic tables and the electronic calculators, the logarithmic values are represented in the form of a finite decimal expression. This may generate the false impression that they are necessarily rational numbers. Unlike many texts and articles on the subject, we are not restricted to the decimal logarithm. In fact, we show a sufficient condition for the $\log b$ number to be irrational, where $a>0$ and $b>1$ are integers and also a criterion that establishes the irrationality of $\log b$ when $b>1$ is square free. We finally show, using GelfondSchneider's Theorem, some proof of the transcendence of certain logarithms, and we deal with logarithms of non-integer real numbers.

Keywords: irrational numbers, logarithms, Fundamental Theorem of Arithmetic, transcendent numbers. 


\section{Introdução e primeiro exemplo}

Os logaritmos surgem desde o Ensino Médio como log 2, por exemplo. Tais números aparecem nas calculadoras eletrônicas, ou mesmo nas antigas tábuas de logaritmos, na forma de uma expressão decimal finita. Isso pode levar um aluno de Ensino Medio a concluir erroneamente que se tratam de números racionais. Como podemos estabelecer a irracionalidade de certos logaritmos? Em geral não é tão simples examinar a natureza (quanto a racionalidade ou não) de um número real. Veremos como o Teorema Fundamental de Aritmética (um resultado acessível ao aluno de Ensino Médio) nos ajuda a investigar a irracionalidade de logaritmos. Antes, no entanto, não é demais relembrar algumas definições.

Sejam $a$ e $b \neq 0$ dois números inteiros. Dizemos que $b$ divide $a$ quando existe um inteiro $c$ tal que $a=b c$. Neste caso, dizemos também que $b$ é um divisor de $a$ ou que $a$ é um múltiplo de $b$.

Todo inteiro positivo a diferente de 1 possui ao menos dois divisores positivos, já que 1 e $a$ são divisores de $a$. Um número inteiro $p>1$ é dito primo quando possui exatamente dois divisores positivos. Um inteiro maior do que 1 que não é primo é dito composto.

Teorema 1 (Teorema Fundamental da Aritmética). Seja a um inteiro diferente de 1, -1 e 0 . Então existem números primos $p_{1}, p_{2}, \ldots, p_{r}$ e inteiros positivos $\alpha_{1}, \alpha_{2}, \ldots, \alpha_{r}$ tais que $a=$ $\pm p_{1}^{\alpha_{1}} p_{2}^{\alpha_{2}} \ldots p_{r}^{\alpha_{r}}$. Além disso, esta decomposição é única, a menos de ordem dos fatores.

Apresentamos, no Exemplo 1, uma aplicação do teorema acima, também conhecido como Teorema da Fatoração Única. Para isso recordamos a definição de logaritmo: dado um número real $a>0$, seu logaritmo na base $b>0$, com $b \neq 1$, é o número real $x$ tal que $b^{x}=a$. Denotamos o logaritmo de $a$ na base $b$ o por $\log _{b} a$. Quando $b=10$ o logaritmo é dito decimal e denotamos $\log _{10} a$ simplesmente por $\log a$. Vamos admitir conhecidas as propriedades operatórias dos logaritmos. Ao longo deste artigo, exceto ao comentar o logaritmo natural, tratamos de logaritmos de base inteira.

Exemplo 1. O número $\log 2$ é irracional.

Note inicialmente que $\log 2>\log 1=0$. Por contraposição, podemos supor que existam inteiros positivos $m$ e $n$ tais que $\log 2=\frac{m}{n}$. Assim, pela definição de logaritmo, $2=10^{\frac{m}{n}}$. Elevando ambos os membros a potência $n$ obtemos $2^{n}=10^{m}=2^{m} 5^{m}$. Segue do Teorema Fundamental da Aritmética que $m=0$, o que contraria a nossa suposição inicial. Portanto, $\log 2$ é irracional.

\subsection{Uma generalização}

O ponto crucial no argumento acima foi o fato de 2 e 10 terem fatores primos diferentes. De forma mais precisa, o fator 5 está na decomposição em primos do número 10, mas não está, evidentemente, na decomposição do número 2. Assim, podemos generalizar o argumento do exemplo anterior para investigar a irracionalidade $\operatorname{de} \log _{b} a$, conforme a proposição a seguir.

Proposição 1. Sejam a e b inteiros positivos com $b \geq 2$. Se as decomposições (ou fatorações) em fatores primos de a ou b apresentam pelo menos um fator que não é comum, então $\log _{b} a$ é um número irracional. 
Demonstração. Vamos supor, por contraposição, que existam dois números inteiros positivos $m$ e $n$ tais que $\log _{b} a=\frac{m}{n}$. Assim, $a=b^{\frac{m}{n}} \Leftrightarrow a^{n}=b^{m}$. Da última igualdade concluímos, com o auxílio do Teorema Fundamental da Aritmética, que os números $a$ e $b$ possuem exatamente os mesmos fatores primos.

Será que vale a recíproca da Proposição 1, ou seja, se $\log _{b} a$ for irracional, então as decomposições em fatores primos de $a$ e de $b$ têm, necessariamente, pelo menos um fator primo desigual? Como

$$
\log 20=\log (2 \cdot 10)=\log 2+\log 10=\log 2+1,
$$

segue do Exemplo 1 que $\log 20$ é irracional. No entanto, os números $20=2^{2} \cdot 5$ e $10=2 \cdot 5$ possuem os mesmos fatores primos. Logo, a recíproca da Proposição 1 é falsa.

Vamos mostrar agora que o número $\log _{b} a$, nas condições da Proposição 1, é transcendente. Antes, no entanto, lembramos a definição de números algébricos e de números transcendentes. Um número complexo é dito algébrico quando é raiz de alguma equação polinomial com coeficientes inteiros. Todo número racional $r$ é algébrico já que $r=\frac{m}{n}$, com $m$ e $n \neq 0$ inteiros, é solução da equação $n x-m=0$. Um número complexo que não é algébrico é dito transcendente. Existe uma infinidade (não-enumerável ${ }^{1}$ ) de números reais transcendentes [5]. Por exemplo, os números e e $\pi$ são transcendentes. Figueiredo (2002).

Uma poderosa ferramenta que nos permite verificar a transcendência de diversos números reais é o Teorema de Gelfond-Schneider (provado no ano de 1934 por Gelfond e independentemente por Schneider em 1935) enunciado a seguir e cuja prova pode ver-se em [3] ou [4].

Proposição 2 (Teorema de Gelfond-Schneider). Sejam $\alpha$ e $\beta$ números algébricos. Se $\alpha \neq 0$, $\alpha \neq 1$ e $\beta$ não for um número real racional, então $\alpha^{\beta}$ é transcendente.

Sejam $a$ e $b>1$ inteiros positivos. Vamos supor que existe um número primo que pertence à fatoração de apenas um dos números $a$ ou $b$. Da Proposição 1 concluímos que $\log _{b} a$ é irracional. Pelo fato de ser inteiro, o número $b$ é algébrico. Suponha que $\log _{b} a$ seja também algébrico. Segue da Proposição 2 que $b^{\log _{b} a}$ é transcendente, o que não pode ocorrer pois $b^{\log _{b} a}=a$ e $a$ é algébrico. Portanto, $\log _{b} a$ é transcendente.

Da transcendência de $\log _{b} a$, decorre a sua não construtibilidade com régua e compasso. Com efeito, somente números algébricos de grau ${ }^{2}$ igual a uma potência de 2 são construtíveis com os instrumentos euclidianos, como se pode ver em [2].

O logaritmo natural, isto é, o logaritmo de base $e\left(\log _{e} a=\ln a\right)$ é tão, ou mais, importante que o logaritmo decimal. Aqui, portanto, cabe a seguinte questão: ln 2, por exemplo, é racional ou irracional? Para responder essa pergunta devemos lançar mão da irracionalidade ${ }^{3}$ do número $e^{r}$ quando $r$ é um número racional não nulo. Uma prova deste fato encontra-se em [3]. Suponha, por

\footnotetext{
${ }^{1}$ Um conjunto infinito $A$ é enumerável quando existe uma função bijetiva $f: \mathbb{N} \rightarrow A$. Do contrário, o conjunto é dito não-enumerável. Sabe-se que o conjunto dos números algébricos, a exemplo do conjunto dos números racionais é enumerável, como se pode ver em [1].

${ }^{2}$ Seja $n$ um inteiro positivo. Um número é dito algébrico de grau $n$ quando é raiz de uma equação polinomial de grau $n$ e não é raiz de nenhuma equação de grau menor do que $n$.

${ }^{3} \mathrm{O}$ resultado mais geral, cuja prova encontra-se em [3] e conhecido como Teorema de Lindemann, afirma que $e^{\gamma}$ é transcendente quando $\gamma$ é um algébrico diferente de zero.
} 
contraposição, que $\ln 2=r$, onde $r$ é um número racional não nulo. Daí, $2=e^{r}$, absurdo pois $e^{r}$ é irracional. Portanto, $\ln 2$ é irracional. O argumento acima pode ser estendido para $\ln q$, onde $q$ é um número racional positivo e diferente de 1 . Daí concluímos que $\ln q$ é irracional quando $q$ é um racional diferente de 1.

\section{Um critério para a irracionalidade de $\log _{b} a$}

Voltamos a nossa atenção aos logaritmos de base inteira.

Um número inteiro $b \neq 0$ é dito livre de quadrados ou sem fator quadrático, quando é um inteiro que não é divisível por nenhum quadrado perfeito diferente de 1 .

Por exemplo, o número 18 não é livre de quadrados pois é divisível por $3^{2}$. O mesmo ocorre com o número $24=2^{3} \cdot 3$ que, ao ser divisível por $2^{3}$, também é divisível por $2^{2}$. Por outro lado, o número $42=2 \cdot 3 \cdot 7$ é livre de quadrados.

Segue do Teorema Fundamental da Aritmética que se $b>1$ é um inteiro livre de quadrados então $b=p_{1} p_{2} \ldots p_{r}$ onde $p_{1}, p_{2}, \ldots, p_{r}$ são primos distintos. Abaixo seguem alguns números livres de quadrados: $1,2,3,5,6,7,10,11,13,14,15,17,19,21,22,23,26,29,30,31,33,34,35,37,38,39,41$. Vamos agora estabelecer um critério que decide pela racionalidade de $\log _{b} a$, quando $b>1$ é um inteiro livre de quadrados. O resultado a seguir generaliza a questão da irracionalidade do logaritmo decimal $\log a$, quando $a$ é um inteiro, tratado em [5].

Proposição 3. Seja a um inteiro positivo e $b>1$ um inteiro livre de quadrados. O número $\log _{b} a$ é racional se, e somente se, $a=b^{\alpha}$, onde $\alpha$ é um inteiro não negativo.

Demonstração. Primeiramente devemos observar que $\log a \geq 0$ quando $a>0$ é inteiro. Além disso, $\log 1=0$. Seja $b=p_{1} p_{2} \ldots p_{r}$, onde $p_{1}, p_{2}, \ldots, p_{r}$ são primos distintos. Suponha agora que existem inteiros positivos $m$ e $n$ tais que $\log _{b} a=\frac{m}{n}$. Segue da Proposição 2 que $a$ e $b$ possuem os mesmos fatores primos em suas respectivas fatorações. Portanto, os fatores primos de $a$ são, precisamente, $p_{1}, p_{2}, \ldots, p_{r}$. Assim,

$$
a=p_{1}^{\alpha_{1}} p_{2}^{\alpha_{2}} \ldots p_{r}^{\alpha_{r}} .
$$

Desta forma, $a=b^{\frac{m}{n}}$ e $p_{1}^{\alpha_{1}} p_{2}^{\alpha_{2}} \ldots p_{r}^{\alpha_{r}}=\left(p_{1} p_{2} \ldots p_{r}\right)^{\frac{m}{n}}$. Elevando a $n$ ambos os membros da última igualdade temos: $p_{1}^{n \alpha_{1}} p_{2}^{n \alpha_{2}} \ldots p_{r}^{n \alpha_{r}}=p_{1}^{m} p_{2}^{m} \ldots p_{r}^{m}$ Segue do Teorema Fundamental da Aritmética que $m=n \alpha_{1}=n \alpha_{2}=\cdots=n \alpha_{r}$. Como $n \neq 0$ segue que $\alpha_{1}=\alpha_{2}=\cdots=\alpha_{r}$. Logo, da Equação (1) concluímos que $a=p_{1}^{\alpha_{1}} p_{2}^{\alpha_{1}} \ldots p_{r}^{\alpha_{1}}=\left(p_{1} p_{2} \ldots p_{r}\right)^{\alpha_{1}}=b^{\alpha_{1}}$. A recíproca é imediata. Com efeito, se $a=b^{\alpha}$ onde $\alpha \geq 0$ é um inteiro, então $\log _{b} a=\log _{b} b^{\alpha}=\alpha$.

Em particular, como o número $10=2 \cdot 5$ é livre de quadrados, segue da proposição anterior que, para $a$ inteiro positivo, $\log a$ é racional se, e somente se, $a=10^{n}$ onde $n$ é um inteiro não negativo. Segue que $\log a$ é inteiro ou irracional.

Utilizando este fato podemos rapidamente determinar se um número $\log a$ é irracional, dado o inteiro $a>0$. Por exemplo, $\log 120$ é irracional pois está estritamente entre dois inteiros consecutivos. Com efeito, $2=\log 10^{2}=\log 100<\log 120<\log 1000=\log 10^{3}=3$.

Será que a Proposição 3 continua válida para um inteiro $b>1$ qualquer no lugar de um inteiro livre de quadrados? A resposta é não. Por exemplo, $\log _{4} 8=\frac{3}{2}$. 


\section{Sobre a irracionalidade de logaritmos de números reais}

A questão que se pretende agora responder diz respeito à racionalidade do número $\log _{b} a, \operatorname{com}$ $b>1$ inteiro e $a>0$ real não inteiro. Por exemplo, $\log _{2} \sqrt{2}=\frac{1}{2}$ é racional e $\log _{2} \sqrt{3}=\frac{1}{2} \log _{2} 3$ é irracional pela Proposição 1. Assim, se a não é inteiro $\log _{b} a$ tanto pode ser racional como irracional.

A resposta fornecida acima não esgota o assunto proposto nesta seção. De fato, os números $\sqrt{2} \mathrm{e}$ $\sqrt{3}$ são ambos irracionais algébricos. Assim, pode-se formular a seguinte pergunta: o que se pode dizer sobre a racionalidade de logaritmos (de base inteira) de números irracionais transcendentes ou de números racionais (não-inteiros)? Começamos respondendo a questão da irracionalidade do número $\log _{b} t$, onde $b>1$ é um inteiro e $t>0$ é transcendente. Para isso utilizamos o corolário a seguir.

Corolário 1. Seja t um número transcendente e $n$ um inteiro positivo. Então a potência $t^{n}$ é um número transcendente.

Demonstração. Vamos supor que $t^{n}$ é um número algébrico. Então ele é raiz da equação polinomial $c_{m} x^{m}+c_{m-1} x^{m-1}+\cdots+c_{1} x+c_{0}=0$, com $m>0$ inteiro e os coeficientes $c_{m} \neq 0, c_{m-1}, \ldots, c_{1}$ e $c_{0}$ números inteiros. Logo,

$$
0=c_{m}\left(t^{n}\right)^{m}+c_{m-1}\left(t^{n}\right)^{m-1}+\cdots+c_{1} t^{n}+c_{0}=c_{m} t^{n m}+c_{m-1} t^{n(m-1)}+\cdots+c_{1} t^{n}+c_{0} .
$$

Portanto, $t$ é solução da equação polinomial $c_{m} x^{n m}+c_{m-1} x^{n(m-1)}+\cdots+c_{1} x^{n}+c_{0}=0$ com coeficientes inteiros. Ou seja, $t$ é um número algébrico.

Agora já estamos em condições de provar que o número $\log _{b} t$ é irracional, onde $b>1$ é um número inteiro e $t>0$ é transcendente. Provemos por redução ao absurdo. Suponhamos que existam inteiros positivos $m$ e $n$ tais que $\log _{b} t=\frac{m}{n}$. Segue que $b^{\frac{m}{n}}=t$ e, portanto,

$$
b^{m}=t^{n} .
$$

Como $t$ é transcendente, segue do Corolário 1 que $t^{n}$ é transcendente. Por outro lado, o número $b^{m}$ é algébrico, pois o conjunto dos números algébricos é fechado em relação a operação de multiplicação como se pode ver em [1]. Logo, a igualdade (2) não pode ocorrer. Assim o número $\log _{b} t$ é irracional.

Nesta seção, tratamos de logaritmos (de base inteira) de números algébricos irracionais e de números transcendentes. Já nas seções anteriores, tratamos de logaritmos de números inteiros. Agora nos resta investigar a natureza dos logaritmos (de base inteira) de números racionais não-inteiros. Para isso, sejam $m$ e $n>1$ inteiros positivos (primos entre si) e $b>1$ um número inteiro. Vamos supor que o número $\log _{b} \frac{m}{n}$ seja racional. Portanto, existem inteiros $p$ e $q>0$, tais que

$$
\log _{b} \frac{m}{n}=\frac{p}{q}
$$

Vamos dividir a nossa argumentação em dois casos. Suponha, primeiramente que $p>0$. Decorre da igualdade (3) que: $b^{\frac{p}{q}}=\frac{m}{n} \Rightarrow n b^{\frac{p}{q}}=m \Rightarrow n^{q} b^{p}=m^{q}$. Segue do Teorema Fundamental da Aritmética que todo fator primo de $n$ é também fator primo de $m$. No entanto, isso não pode 
ocorrer pois os números $m$ e $n>1$ são, por hipótese, primos entre si. Portanto, o número $\log _{b} \frac{m}{n}$ é irracional.

Suponha agora que $p<0$. Da igualdade (3) temos que:

$$
b^{\frac{p}{q}}=\frac{m}{n} \Rightarrow n b^{\frac{p}{q}}=m \Rightarrow n^{q} b^{p}=m^{q} \Rightarrow n^{q}=m^{q} b^{-p} \Rightarrow n^{q}=m^{q} b^{p_{1}}, \text { onde } p_{1}=-p>0 .
$$

Do Teorema Fundamental da Aritmética e da última igualdade acima, concluímos que todo fator primo de $m$ é também fator primo de $n$. No entanto, $m$ e $n$ são primos entre si. Assim, concluímos que $m=1$ e que $n^{q}=b^{p_{1}}$. Em palavras, $n^{q}$ é uma $p_{1}$-ésima potência de $b$. Portanto, esta é a única situação em que $\log _{b} \frac{m}{n}$ é um número racional.

Por exemplo, o número $\log _{4} \frac{1}{8}=-\frac{3}{2}$ é racional. Note que $8^{2}=4^{-(-3)}$. Isso completa o nosso estudo quanto a irracionalidade de um logaritmo de base inteira de um número racional não inteiro.

\section{Resultados adicionais}

Vamos apresentar, nesta seção, alguns problemas de logaritmos decimais cuja solução recaem no Teorema Fundamental da Aritmética ou no Teorema de Gelfond-Schneider. Comecemos com a seguinte indagação: o número $\frac{\log 3}{\log 2}$ é racional ou irracional? O resultado a seguir responde a esta questão.

Proposição 4. Sejam a e $b \neq 1$ inteiros positivos tais que as suas decomposições (ou fatorações) em fatores primos apresentam pelo menos um primo que não é comum. Então o número $\frac{\log a}{\log b}$ é irracional.

Demonstração. Basta notar que $\frac{\log a}{\log b}=\log _{b} a$ e utilizar a Proposição 1 para obter a tese.

A proposição a seguir nos diz que o número $\frac{\log 3}{\log 2}$, além de irracional, é transcendente.

Proposição 5. Sejam $a, b>0$ números reais algébricos com $b \neq 1$. O número real $\frac{\log a}{\log b}$ é racional ou transcendente.

Demonstração. Suponha que $\frac{\log a}{\log b}=\beta$, onde $\beta>0$ é um irracional algébrico. Com isso,

$$
a=b^{\beta} .
$$

Como $b \neq 1$ é positivo e $\beta$ é, por hipótese, um irracional algébrico, segue da Proposição 3 que $b^{\beta}$ é transcendente. Por outro lado, $a$ é algébrico, o que origina uma contradição em virtude da igualdade (4). Portanto, o número $\frac{\log a}{\log b}$ é transcendente. 
Segue do resultado anterior que o número $\frac{\log a}{\log b}$, nas condições da Proposição 4, é transcendente.

Sejam $a$ e $b$ dois números reais positivos. Dizemos que $\log a$ e $\log b$ são linearmente independentes sobre $\mathbb{Q}$ quando a equação $r \log a+s \log b=0$, com $r$ e $s$ racionais, implica em $r=s=0$. Do contrário, dizemos que são linearmente dependentes sobre $\mathbb{Q}$.

Vamos denotar por $\mathcal{A}$ o conjunto dos números reais algébricos.

Proposição 6. Sejam a e b números reais algébricos positivos. A independência linear de $\log a$ e $\log b$ sobre $\mathbb{Q}$ implica na independência linear sobre $\mathcal{A}$.

Demonstração. Suponha que que $a, b, \alpha$ e $\beta$ são números algébricos tais que $\alpha \log a+\beta \log b=0$. Daí segue que $\frac{\log a}{\log b}=-\frac{\beta}{\alpha}$. Como $\alpha$ e $\beta$ são algébricos, então $-\frac{\beta}{\alpha}$ também é um número algébrico [1]. Como não é transcendente, segue da Proposição 5 que $\frac{\log a}{\log b}=r$, onde $r$ é um número racional. Portanto, $\log a=r \log b \Rightarrow \log a-r \log b=0$. Ou seja, $\log a$ e $\log b$ são linearmente dependentes sobre $\mathbb{Q}$.

Como $\mathbb{Q}$ é um subconjunto de $\mathcal{A}$ então a independência linear de $\log a$ e $\log b$ sobre $\mathcal{A}$ implica na indepencência sobre $\mathbb{Q}$. Ou seja, a recíproca da proposição anterior é verdadeira.

Observação 1. A Proposição 6 é equivalente ao Teorema de Gelfond-Schneider, provado no ano de 1934. Ela nos diz que se $a>0, b>0, \alpha$ e $\beta$ são números reais algébricos, $\operatorname{com} \log a$ e $\log b$ linearmente independente sobre $\mathbb{Q}$, então $\alpha \log a+\beta \log b \neq 0$. No ano de 1966 o matemático inglês Alan Baker provou que este resultado continua verdadeiro para uma quantidade arbitrária de logaritmos. Essa prova lhe rendeu a Medalha Fields em 1970 ([3]).

\section{Conclusão}

Os exemplos de irracionalidade no Ensino Médio normalmente se restringem somente a poucos números como $\sqrt{2}$, o Número de Ouro $\phi$ ou $\pi$, além de números cuja expressão decimal é fornecida. No entanto, a demonstração da irracionalidade do número $\pi$ requer conhecimentos de Cálculo. Assim apresentamos outros exemplos de números irracionais que surgem para alunos do Ensino Médio (os logaritmos) e cuja prova é acessível já que utiliza somente o Teorema Fundamental da Aritmética.

\section{Referências}

[1] Figueiredo, Djairo Guedes. Números Irracionais e Transcendentes. Rio de janeiro: SBM, 2002.

[2] Herstein, I. N. Topics in Algebra. 2. ed. Wiley India Pvt. Limited, 2006.

[3] Marques, Diego. Teoria dos números transcendentes. Rio de Janeiro: SBM, 2013.

[4] Niven, Ivan. Irrational numbers. Washington D.C. Mathematical Association of America, 1967.

[5] Niven, Ivan. Numbers: Rational and Irrational. Washington D.C. Mathematical Association of America, 1961. 
Ronald Pinto

Colégio Pedro II

$<$ ronaldsimoes@gmail.com>

Liliana da Costa

Colégio Pedro II

<lmgccosta@gmail.com>

Recebido: 25/06/2018 